\title{
Epithelial self-defense against cancer
}

\author{
Hajime Yamauchi ${ }^{1}$, Yasuyuki Fujita ${ }^{1}$ \\ ${ }^{1}$ Division of Molecular Oncology, Institute for Genetic Medicine, Hokkaido University, North 15 West 7, Kita-ku, Sapporo, Hok- \\ kaido 060-0815, Japan \\ Cell Research (2012) 22:1527-1529. doi:10.1038/cr.2012.69; published online 24 April 2012
}

It is not clearly understood what happens at the interface between normal and transformed epithelial cells at the first step of carcinogenesis. A recent study reveals that the organized epithelial structure suppresses clonal expansion of transformed cells. Translocation from the epithelium or perturbation of intercellular adhesions may be required for transformed cells to evade the suppressive environments.

During the initial stage of carcinogenesis, transformation occurs in a single cell within an epithelial monolayer [1, 2]. However, it is not clearly understood what happens at the interface between normal and transformed cells during this process. Are those transformed cells always accumulated in epithelia or are they eliminated from normal epithelial environments? In Drosophila melanogaster, normal and transformed cells often compete with each other for survival. For example, when Lethal giant larvae $(L g l)$-knockout cells are surrounded by wild-type cells, $L g l$-knockout cells die by apoptosis [3, 4]. When Src-activated cells are surrounded by wild-type cells, Src-activated cells are basally extruded and leave the epithelium [5]. These phenomena are called 'cell competition', which has been intensively studied in

Correspondence: Yasuyuki Fujita

Tel: +81-11-706-5530; Fax: +81-11-706-7544

E-mail: yasu@igm.hokudai.ac.jp
Drosophila [6-8]. However, it was not known whether comparable phenomena also occur in mammals.

Recent studies using mammalian cell culture systems have revealed that various phenomena can occur at the interface between normal and transformed epithelial cells [4, 9-11]. In these studies, the authors have established Madin-Darby canine kidney (MDCK) epithelial cell lines expressing oncoproteins or shRNA of tumor suppressor proteins in a tetracycline- or temperature-inducible manner. First, normal and transformation-inducible cells are mixed, and incubated until the mixture of cells form a monolayer of epithelial sheets. Transformation is then induced by addition of tetracycline or temperature shift, and the fate of transformed cells surrounded by normal cells is examined. When oncoprotein $\mathrm{Ras}^{\mathrm{V} 12}$ - or $\mathrm{v}$-Src-transformed cells are surrounded by normal epithelial cells, the transformed cells are apically extruded and leave the epithelium $[9,10]$. Interestingly, during these processes, a number of molecules or signaling pathways including mitogen-activated protein kinase (MAPK) and myosin-II are activated in the transformed cells surrounded by normal cells. And activation of some of those molecules has been shown to play a crucial role in apical extrusion of the transformed cells. In addition, when tumor suppressor protein Lgl- or Mahjong-knockdown cells are surrounded by normal epithelial cells, the knockdown cells undergo apoptosis and are eliminated from the epithelium [4, 11]. c-Jun NH2-terminal kinase (JNK) or p38MAPK plays a crucial role in apoptosis of Lgl- or Mahjongknockdown cells, respectively. Importantly, when the transformed cells alone are present, neither apical extrusion nor apoptosis occurs. Collectively, these data indicate that the presence of surrounding normal epithelial cells substantially influences the signaling pathways and fate of transformed cells and that the epithelium has an ability to exclude transformed cells from their society $[12,13]$.

In a recent study published in Nature, Leung and Brugge further developed the notion that the epithelium provides suppressive environments against tumor formation [14]. In this study, they used three-dimensional (3D) organotypic cultures using MCF10A cells, a nontransformed human mammary epithelial cell line. When cultured in Matrigel that is composed of a mixture of extracellular matrix proteins, MCF10A cells formed polarized acinar structures containing an internal lumen, and eventually ceased proliferation. By infecting the acini with low-dose lentiviral vectors, Leung and Brugge established experimental conditions where oncoproteins are sporadically expressed in single cells. The fate of these single mutant cells in organotypic mammary acini was then analyzed by long-term microscopic observations. 
ERBB2 is an oncogenic receptor tyrosine kinase and is overexpressed in $30 \%$ of breast cancers. When ERBB2 was overexpressed in sporadic cells within 3D acini, ERBB2-overexpressing cells were translocated into the lumen, followed by clonal expansion. The appearance of the luminal outgrowth of the transformed cells resembles that of early-stage ductal carcinoma in situ (DCIS), but differs in that no ERBB2overexpressing cells remain within the epithelium as the DCIS tumor cells grow by replacing normal epithelia. Therefore, it is not clear at present whether the observed translocation of ERBB2-overexpressing cells reflects tumor formation or extrusion from the epithelium. Leung and Brugge presented data indicating that the MAPK pathway is involved in luminal translocation of ERBB2-overexpressing cells. Interestingly, MAPK has also been shown to play a crucial role in apical extrusion of $\mathrm{Ras}^{\mathrm{V} 12}$ - or v-Src-transformed epithelial cells that are surrounded by normal epithelial cells $[9,10]$. Thus, MAPK seems to be a general regulator for apical extrusion of transformed cells from the epithelium. Furthermore, Leung and Brugge showed that inhibition of matrix metalloproteinases (MMPs) substantially blocks ERBB2-mediated luminal translocation. Surprisingly, sporadic overexpression of MT1-MMP promoted translocation to the lumen, and weakening of the cell-matrix adhesion was likely to be involved in this process. However, translocated MT1-MMP-overexpressing cells did not proliferate in the lumen, suggesting that translocation alone is not sufficient to facilitate clonal expansion. This is the first demonstration that MMP can play a crucial role in apical extrusion of transformed cells from the epithelium.

Myc is a transcriptional factor whose overexpression often causes overproliferation of cells and is found in many types of cancers. However, when Myc was sporadically expressed in single cells in 3D acini, Myc-overexpressing cells did not proliferate and stayed as single cells within the epithelial layer. Similarly, when constitutively active AKT (myrAKT1) was expressed in single cells, myrAKT1-expressing cells remained quiescent in the epithelial layer. In addition, MMP inhibitortreated ERBB2-overexpressing cells stayed in the epithelial layer and were unable to proliferate. These data indicate that epithelial environments have suppressive roles in proliferation of transformed cells. Leung and Brugge then examined whether cell displacement from the epithelium facilitates the clonal outgrowth of sporadic mutant cells by using MT1-MMP as a tool to drive cell translocation. When MT1MMP and myrAKT were coexpressed in single cells, the mutant cells were translocated to the lumen and proliferated. In contrast, coexpression of MT1-MMP and Myc induced luminal translocation, but clonal expansion did not occur due to increased apoptosis. Collectively, these results demonstrate

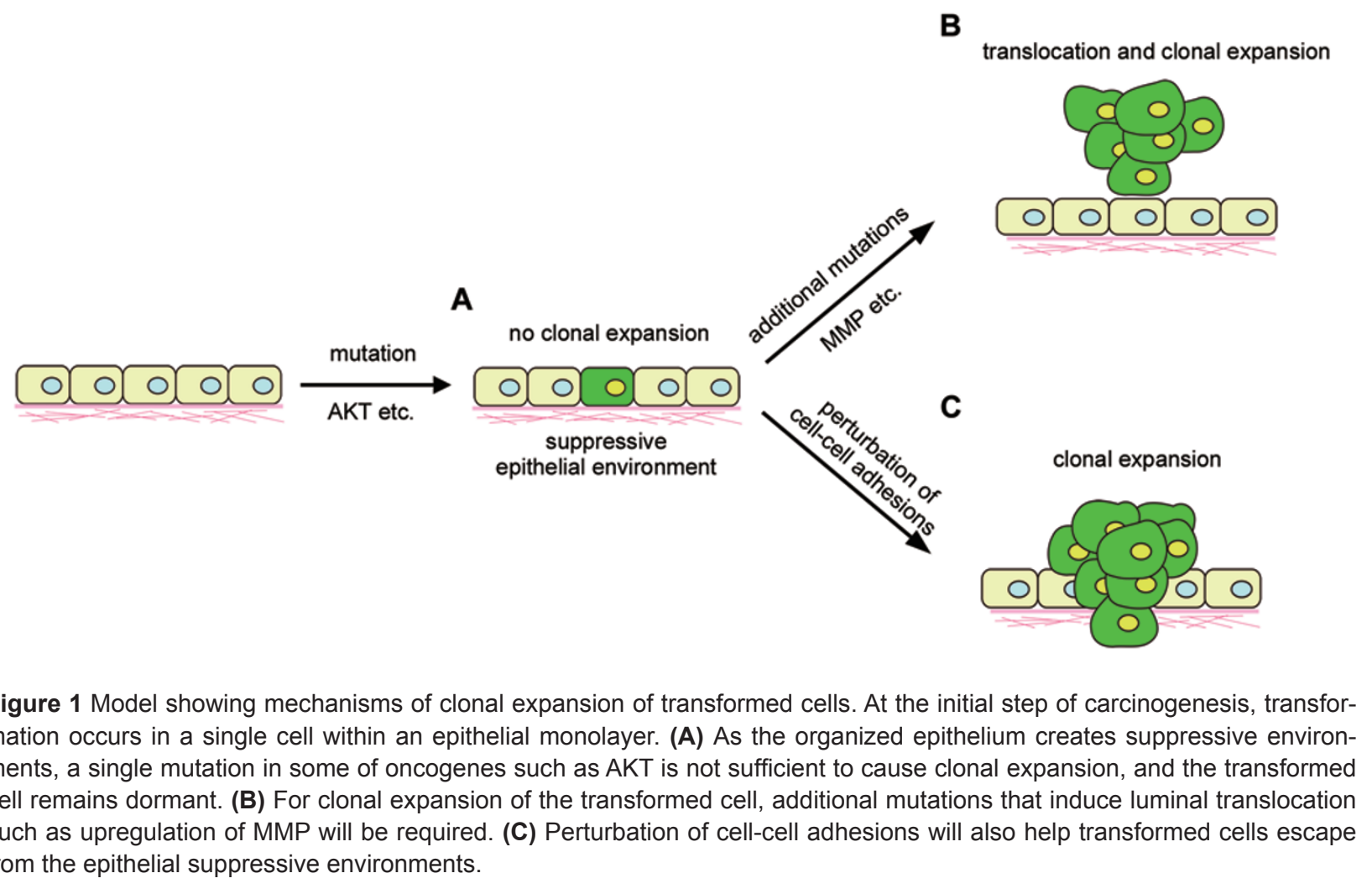

Cell Research | Vol 22 No 11 | November 2012 
that cell translocation allows sporadic mutant cells to evade suppressive environments and elicits clonal selection depending on which signaling pathways are dysregulated in the mutant cells.

In epithelial sheets, each epithelial cell is connected with each other via Ecadherin-based intercellular adhesions. Leung and Brugge examined whether E-cadherin-based cell-cell contacts have a role in suppressive epithelial environments, by knocking down p120-catenin, a crucial regulator of the E-cadherin complex. In p120-catenin-knockdown acini, Myc- or myrAKT-overexpressing cells underwent clonal expansion without luminal translocation, suggesting that the epithelial organization mediated by E-cadherin-based cell-cell contacts is crucial for suppressing proliferation of mutant cells.

The study by Leung and Brugge clearly demonstrates that the proper epithelial structure provides suppressive environments against clonal expansion of transformed cells. According to their results, one can postulate that transformed cells may remain dormant while being surrounded by normal cells within epithelium (Figure 1A). For clonal expansion of the transformed cells, additional mutations that induce luminal translocation such as upregulation of MMP will be required (Figure 1B). Pathological conditions that perturb intercellular adhesions of epithelia (e.g., chronic infection or inflammation) may also help transformed cells escape from the proliferative constraint (Figure 1C). What remains unknown is the molecular mechanism that creates epithelial suppressive environments. Recent Droso- phila studies have shown that normal and transformed cells can recognize the difference(s) between them during cell competition. For example, a membrane protein Flower has been shown to play a role in intercellular recognition between normal and dMyc-overexpressing cells [15]. It remains to be studied whether a comparable molecular mechanism is also involved in cell competition in mammals. It is expected that future studies will reveal a number of molecules that play a crucial role in cell-cell recognition and downstream signaling pathways at the interface between normal and transformed epithelial cells. By further investigation, we may be able to establish novel types of cancer prevention and treatment: enhancing the ability of surrounding normal cells to fight against cancer cells or attenuating the defense of cancer cells against neighboring normal cells.

\section{References}

1 Fialkow PJ. Clonal origin of human tumors. Biochim Biophys Acta 1976; 458:283-321.

2 Nowell PC. The clonal evolution of tumor cell populations. Science 1976; 194:23-28

3 Grzeschik NA, Amin N, Secombe J, Brumby AM, Richardson HE. Abnormalities in cell proliferation and apico-basal cell polarity are separable in Drosophila lgl mutant clones in the developing eye. Dev Biol 2007; 311:106123.

4 Tamori Y, Bialucha CU, Tian AG, et al. Involvement of $\mathrm{Lgl}$ and Mahjong/ VprBP in cell competition. PLoS Biol 2010; 8:e1000422.

5 Vidal M, Larson DE, Cagan RL. Csk- deficient boundary cells are eliminated from normal Drosophila epithelia by exclusion, migration, and apoptosis. Dev Cell 2006; 10:33-44.

6 Baker NE, Li W. Cell competition and its possible relation to cancer. Cancer Res 2008; 68:5505-5507.

7 Diaz B, Moreno E. The competitive nature of cells. Exp Cell Res 2005; 306:317-322.

8 Johnston LA. Competitive interactions between cells: death, growth, and geography. Science 2009; 324:1679-1682.

9 Hogan C, Dupré-Crochet S, Norman $\mathrm{M}$, et al. Characterization of the interface between normal and transformed epithelial cells. Nat Cell Biol 2009; 11:460-467.

10 Kajita M, Hogan C, Harris AR, et al. Interaction with surrounding normal epithelial cells influences signalling pathways and behaviour of Src-transformed cells. J Cell Sci 2010; 123:171180.

11 Norman M, Wisniewska KA, Lawrenson $\mathrm{K}$, et al. Loss of Scribble causes cell competition in mammalian cells. $J$ Cell Sci 2012; 125:59-66.

12 Hogan C, Kajita M, Lawrenson K, Fujita Y. Interactions between normal and transformed epithelial cells: Their contributions to tumourigenesis. Int $J$ Biochem Cell Biol 2011; 43:496-503.

13 Fujita Y. Interface between normal and transformed epithelial cells-A road to a novel type of cancer prevention and treatment. Cancer Sci 2011; 102:17491755.

14 Leung CT, Brugge JS. Outgrowth of single oncogene-expressing cells from suppressive epithelial environments. Nature 2012; 482:410-413.

15 Rhiner C, López-Gay JM, Soldini D, et al. Flower forms an extracellular code that reveals the fitness of a cell to its neighbors in Drosophila. Dev Cell 2010; 18:985-998. 